\title{
Mieux prendre en compte l'usager dans le design de transport public Cas d'étude sur les navettes autonomes à Sion
}

\author{
Randolf Ramseyer ${ }^{1}$, Emmanuel Fragnière ${ }^{1,2}$, Benjamin Nanchen ${ }^{1}$, \\ Florian Evéquoz ${ }^{1,3}$, Grace Eden ${ }^{4}$ \\ ${ }^{1}$ University of Applied Sciences Western Switzerland (HES-SO, Sierre, Switzerland) \\ ${ }^{2}$ University of Bath (Bath, UK) \\ ${ }^{3}$ University of Fribourg (Fribourg, Switzerland) \\ ${ }^{4}$ Manchester Metropolitan University (Manchester, United Kingdom) \\ randolf.ramseyer@hevs.ch \\ $<$ www.hevs.ch/servicedesign >
}

\section{Résumé}

Dans le domaine de la mobilité, l'arrivée de systèmes de transport autonome prend de l'ampleur. Dès lors, il est capital de prendre en compte les facteurs qui auront une incidence sur l'adoption de ces systèmes par les utilisateurs. De manière générale, pour que la mise en œuvre d'une technologie soit réussie, le service fourni par celle-ci doit améliorer le confort et la sécurité de l'utilisateur. Nous présentons dans cet article les résultats d'immersion de 21 utilisateurs de la navette autonome de la ville de Sion en Suisse. Cette étude est la première étape d'une approche de design de service afin d'optimiser les navettes autonomes en tant que service public de mobilité.

\section{Mots-clés}

Véhicule autonome, perception, usage, risque, mobilité

\begin{abstract}
Taking better account of the user in the design of public transport. Case study on autonomous shuttles in Sion - In the field of mobility, the arrival of autonomous transport systems is increasing. It is therefore essential to take into account the factors that will have an impact on the adoption of these systems by users. In general, for the implementation of a technology to be successful, the service provided by it must improve the comfort and safety of the user. In this article we present the immersion results of 21 users of the autonomous shuttle from the city of Sion in Switzerland. This study is the first step in a service design approach to optimize autonomous shuttles as a public mobility service.
\end{abstract}

\section{Keywords}

Autonomous vehicle, perception, uses, risk, mobility

\section{Sommaire / Content}

1. Contexte

2. Etat de l'art

3. Cas d'étude sur la navette autonome

4. Analyse des données

5. Discussion

6. Conclusion

Bibliographie 


\section{Ocula ${ }^{20}$}

\section{Contexte}

La vision des villes de demain est fortement liée à celle de la configuration future de leurs systèmes de transport. L'augmentation des voitures privées a fortement affecté l'aménagement de régions périphériques de faible densité et dispersées. Celle-ci a eu un impact négatif sur la qualité de vie dans les villes en générant plus de trafic, une rarification des places de stationnement et un accroissement des émissions de gaz polluant néfastes pour la santé (cf. Alessandrini et al. 2015). L'introduction de véhicules autonomes dans l'espace public soulève de multiples questions. Ces technologies progressent rapidement et induisent des changements profonds en terme de mobilité urbaine (cf. Nicolas et al. 2016). Face à des logiques de rupture radicale de ce type, il est nécessaire de faire preuve d'innovation dans les services. Dans le cas particulier des entreprises produisant des biens, cela passe traditionnellement par la recherche et développement, fonction souvent appelée R\&D. Dans le domaine des services, la R\&D est plus compliquée à aborder puisque la production et la consommation se produisent simultanément et impliquent le consommateur en tant co-créateur de valeur (cf. Lusch 2004).

Le lancement d'une nouvelle navette autonome en tant que service dans l'espace public est un défi. Comment les utilisateurs l'accepteront-ils ? Quels sont les risques perçus ? Quel est leur sentiment général au sujet de cette nouvelle technologie ? Répondre à ces questions aidera les entreprises technologiques ainsi que les entreprises de services à lancer une nouvelle technologie en tant que service.

\subsection{Véhicules Autonomes}

Un véhicule peut être automatisé à différents degrés. La conduite autonome représente son degré le plus avancé (cf. Florez et al. 2018). A Sion (Suisse), pour la première fois dans le monde, des navettes autonomes sont utilisées sur la voie publique. Deux trajets sont proposés : un pour relier la gare au centre-ville de Sion et l'autre pour effectuer un parcours dans la vielle ville. Ces navettes autonomes électriques roulent à une vitesse maximale de 20 kilomètres à l'heure et peuvent transporter 11 passagers (cf. Eden et al. 2017). Pour ce test à l'échelle $1: 1$, un accompagnant (un groom) est responsable de la sécurité et de la surveillance du système. Ce projet (« postauto.ch $2016 »$ ) associe la société de transport (La Poste), les pouvoirs publics (Ville de Sion et Canton du Valais) et les Universités (EPFL et HES-SO Valais-Wallis). La première phase de ce test s'est déroulée dans la vieille ville de Sion sur un parcours de 1,5 $\mathrm{km}$ (zones pour véhicules et piétons). L'itinéraire a ensuite été prolongé sur une route plus dense, équipée de feux de circulation intelligents capables de communiquer avec les navettes autonomes et franchissant un rond-point. 


\section{Ocula ${ }^{20}$}

La place de l'usager en design

R. Ramseyer, E. Fragnière, B. Nanchen, F. Evéquoz, G. Eden I Mieux prendre ... I DOI: 10.12977/ocula2019-5

\subsection{La navette autonome en tant que service de transport public}

Dans le secteur de la mobilité, un grand nombre de nouvelles technologies et de services sont en train d'émerger. L'introduction de véhicules autonomes en tant que service de transport public implique non seulement les usagers ou passagers, mais aussi les autorités, les fabricants, les sociétés de transport public, les agents de la loi, les conducteurs, les piétons et les commerçants. Certains auteurs (cf. Fragnière et al. 2017) définissent le service comme une résolution de problème qui simplifie la vie de l'utilisateur et en même temps ajoute de la valeur.

\subsection{Le Service Design comme approche R\&D Service pour l'implication en amont de l'utilisateur}

Notre approche R\&D Service se fonde sur une méthodologie de Design de service élaborée par l'Institut d'Entrepreneuriat \& Management de la Haute Ecole Spécialisée de Suisse occidentale (HES-SO Valais-Wallis). Elle nous permet de mieux comprendre l'expérience des utilisateurs ainsi que l'analyse de leurs comportements, de même que ceux des autres individus impactés par la navette. Le cas des navettes autonomes de Sion est particulièrement intéressant puisqu'il représente un projet pilote d'où découlera des préceptes généraux pour améliorer ce type de mobilité. Pour ce faire, nous avons impliqué des utilisateurs tests (21 au total) en immersion afin de collecter leurs observations et leurs ressentis. Ainsi, Les données récoltées durant le test à l'échelle $1: 1$ du service de mobilité proposé par la navette autonome en Ville de Sion ont ainsi apporté aux différentes équipes de projet des informations relatives à la perception de l'usager comme par exemple l'utilité, l'efficacité, la sécurité, le confort et l'utilisabilité. De plus, les résultats dévoilent de nouveaux éclairages, notamment des liens problématiques entre l'expérience utilisateur, la perception du risque et l'acceptabilité d'une nouvelle technologie. Seuls les aspects liés aux à "l'expérience » de la navette autonome ont été retenus dans le cadre de cette étude. En effet, les éléments liés aux attentes générales des utilisateurs quant aux véhicules autonomes n'ont pas été abordés. Enfin, par rapport à notre approche de design de service, cette étude se concentre essentiellement sur l'identification de ces différents attributs. Ces derniers seront exploités dans le cadre d'une recherche future pour améliorer les « modes opératoires » de ce nouveau type de service de mobilité.

\section{Etat de l'art}

La technologie est en phase de devenir un substitut de plus en plus courant pour remplacer le travail de l'humain. Des tâches qui nécessitaient autrefois un travail réfléchi et minutieux par l'esprit humain sont exécutées par des machines sophistiquées. Pendant que les ingénieurs résolvent de nouveaux défis liés à une telle technologie, d'importants obstacles psychologiques auxquels les utilisateurs devront faire face lorsqu'ils utiliseront cette technologie émergent (cf. Waytz et al. 2014). Toutefois, en dépit de leur utilisation croissante, 


\section{Ocula ${ }^{20}$}

La place de l'usager en design

R. Ramseyer, E. Fragnière, B. Nanchen, F. Evéquoz, G. Eden I Mieux prendre ... I DOI: 10.12977/ocula2019-5

ces applications suscitent toujours un certain scepticisme au sein de la société (cf. Hengstler et al. 2016). Pour les véhicules entièrement autonomes, bien que les potentiels soient énormes, la confiance nécessaire pour adopter cette technologie n'est pas encore au rendez-vous et il est essentiel de la renforcer (cf. Abraham et al. 2017).

L'évolution des transports publics sans conducteur à l'échelle mondiale, peut amener une vraie révolution en matière de mobilité urbaine. Des données empiriques sur l'expérience des utilisateurs sont essentielles, car le sentiment de sécurité et de sûreté de l'usager a une influence significative au final sur son acceptation. Par contre, celles-ci sont encore insuffisantes (cf. Salonen 2018).

Selon Dubois et al. (2009), l'intégration des notions d'usage et d'usager dans l'acceptabilité des technologies a permis de changer cette vision un peu trop réductrice de l'utilisateur passif. Puisant leurs paradigmes dans des disciplines variées, les études effectuées sur les usages se fondent sur des disciplines autant scientifiques que philosophiques telles que la psychologie, la sociologie, l'anthropologie ou encore la phénoménologie pour n'en citer que quelques-unes (cf. Proulx 2015). Avec pour principe d'intégrer l'usager final au centre du design, cette approche s'articule autour de différentes techniques de collecte de données comme par exemple la simulation, le banc d'essai, l'observation, et les enquêtes de terrain.

\subsection{Méthodologie du Service Design Lab}

La méthodologie de Service Design élaborée et testée par de l'Institut d'Entrepreneuriat \& Management de la HES-SO Valais-Wallis comprend quatre étapes : exploration, script, mise en scène du service et production. Celles-ci permettent de concevoir et d'améliorer des services qui répondent aux besoins et aux attentes des clients. Selon Mager (2008) et le rapport du UK Design Council (2015), le double objectif du Design de service est de s'assurer que, du point de vue du client, l'interface de service est utile, utilisable et souhaitable, et que du point de vue du fournisseur celle-ci soit efficiente, efficace, effective et différenciatrice. Grâce à une approche innovante et itérative basée sur la théâtralisation, les services sont mis en scène afin de tester, d'améliorer et d'évaluer leur qualité. Il est alors possible de découvrir les informations sur les besoins des usagers et les conditions dans lesquelles le service sera exploité. Le niveau de détail augmentera au fur et à mesure.

\subsubsection{Exploration}

La phase d'exploration, basée sur des méthodologies de recherche qualitative éprouvées, permet l'étude descriptive et analytique du terrain, du service et du problème (cf. Crewsell 2012). Les approches telles que l'ethnométhodologie, l'éthologie ou la phénoménologie sont réalisées au moyen d'entretiens semi-directifs, d'entretiens de groupes (focus group), d'immersions, d'expérimentations sociales et de benchmarking. L'étude présente repose essentiellement sur cette première étape du design de service. 


\section{Ocula ${ }^{20}$}

La place de l'usager en design

R. Ramseyer, E. Fragnière, B. Nanchen, F. Evéquoz, G. Eden I Mieux prendre ... I DOI: 10.12977/ocula2019-5

\subsubsection{Script}

L’innovation est la capacité de générer des idées nouvelles et utiles et de les transférer sur le marché. Il s'agit d'une composante essentielle d'un design performant dans l'économie d'aujourd'hui. Chan et al. (2011) ont démontré que la meilleure façon d'innover est de le faire dans la phase d' « idéation », où les concepts et les idées sont générés soit intuitivement, soit par des processus systématiques. Basée sur les données collectées, cette phase d'analyse et de conception, appelé script, utilise des outils de marketing des services, tels que les « Customers Journeys », les « Services Blueprints », l'écriture de scénarios, les «User Stories » et les «personas ». Elle permet une meilleure représentation de l'utilité du service et de ses fonctionnalités pour tangibiliser l'expérience du service.

\subsubsection{Mise en scène du service}

Une fois le service conçu et scénarisé, il peut être prototypé et mis en scène à l'aide d'outils comme la théâtralisation ou encore le jeu de rôle. Un prototype est une visualisation du service contenant une compréhension de ce que la solution devrait être et de ce qu'elle devrait fournir aux utilisateurs, en tant que service (cf. Blomkvist 2014). Les premiers commentaires des utilisateurs peuvent être recueillis pour améliorer le prototype de service et ainsi « rendre visible l'invisible ».

\subsubsection{Production}

Pour permettre aux clients de juger de la qualité du service et de décider de le commercialiser, un test à l'échelle 1 :1 est organisé. Cette phase mesure sa qualité par le retour d'information des usagers, et évalue son prix et la propension à payer, aussi appelée « Willingness To Pay » (cf. Homburg 2005).

\subsection{Fondement scientifique de la méthode}

Cette méthodologie de Service Design est une approche scientifique de conception de service fondée sur des données factuelles (cf. Carr et al. 2011). Ce processus de validation est similaire à la visite d'un patient chez le médecin en trois étapes (cf. Simon 1990) : l'examen, le diagnostic et la prescrition. L'examen, incluant l'anamnèse (analyse de la situation initiale), consiste à recueillir et à comprendre des informations afin d'identifier le problème (connaissances explicites tacites). Chaque élément est mesuré de façon à ce que le problème principal puisse être calculé et défini dans l'énoncé même du problème.

Le diagnostic (inventaire et cartographie, modélisation des problèmes) correspond à la phase d'analyse. Le problème est structuré en effectuant une analyse des sources de contradiction et d'erreur à l'aide des techniques de résolution de problèmes, comme par exemple la matrice des contradictions.

La prescription correspond à la phase d'évaluation de la solution. Les idées générées sont évaluées par ordre de priorité en se basant sur des contraintes locales afin de formuler la solution idéale. Le résultat final consiste en une liste de solutions conceptuelles novatrices possibles. 


\section{Ocula ${ }^{20}$}

La place de l'usager en design

R. Ramseyer, E. Fragnière, B. Nanchen, F. Evéquoz, G. Eden I Mieux prendre ... I DOI: 10.12977/ocula2019-5

Si des solutions ne sont toujours pas trouvées après élimination des contradictions, ou dans le cas où un autre problème survient après l'évaluation de la solution, le processus de résolution des problèmes repart à la première étape afin de redéfinir la situation initiale (processus itératif).

\section{Cas d'étude sur la navette autonome}

A Sion (Suisse), depuis le printemps 2016, une navette autonome est testée à l'échelle $1: 1$. Ce test qui intervient durant l'étape production (cf. 2.2.4), nous permet donc de récolter des données réelles sur la perception qu'on les usagers de cette nouvelle technologie. L'analyse de ces données permet d'amorcer une nouvelle itération (phase d'exploration) de notre méthode de Design de service afin d'améliorer la qualité du service, soit l'utilisation d'une navette autonome comme service de transport public.

A ce titre, nous avons effectué des immersions, des entretiens avec les utilisateurs (passagers, conducteurs de véhicule de tout type, autorités et commerçants) et des observations en milieu naturel afin de recueillir des données. De plus, en avril 2018, 21 étudiants en gestion des risques d'une université suisse (étudiants en mastère) ont utilisé pour la première fois la navette autonome. Afin de récolter leurs perceptions avant, pendant et après cette expérience, les participants devaient répondre à une enquête qualitative sur leurs perceptions au moyen de deux questionnaires qui leur ont été remis avant et après le trajet.

L'analyse de ces données nous permet de mieux comprendre l'acceptation de cette nouvelle technologie par les usagers et de proposer de nouveaux scénarios d'utilisation de la navette autonome répondant mieux à leurs besoins.

\section{Analyse des données}

Nous proposons dans cette section les constats majeurs tirés de l'analyse des transcriptions obtenues à partir des épisodes d’immersion en navette autonome.

Sur la base des entretiens réalisés, l'opinion générale est, au regard des navettes autonomes, très bonne pour l'image de la ville. Les usagers caractérisent aujourd'hui la ville comme une destination innovante.

Les utilisateurs ont emprunté la navette par curiosité et ne l'ont utilisé qu'une seule fois, dans la plupart des cas. L'itinéraire actuel n'est pas pratique. Beaucoup de gens estiment que la navette ralentit la circulation dans le centre-ville : une zone petite et dense.

La navette est considérée comme moyen que les touristes peuvent utiliser pour se familiariser avec la vieille ville.

Plus de la moitié des personnes de notre échantillon ont déclaré qu'elles évitaient complètement les navettes autonomes ou étaient extrêmement prudentes lorsqu'elles marchent ou conduisent près de la navette parce qu'elles avouent avoir des difficultés à interpréter et donc à prédire leurs mouvements (qui sont basés sur des algorithmes). Les citoyens perçoivent également un risque lié aux erreurs potentielles de la technologie. 


\section{Ocula ${ }^{20}$}

La place de l'usager en design

R. Ramseyer, E. Fragnière, B. Nanchen, F. Evéquoz, G. Eden I Mieux prendre ... I DOI: 10.12977/ocula2019-5

De nombreux usagers ont suggéré l'installation d'un dispositif de communication pour mieux informer les autres utilisateurs de la route. Leurs suggestions comprennent également de plus grands indicateurs de signalisation, de sons ou encore un affichage électronique installé directement sur les véhicules.

\section{Discussion}

Nous avons pu constater que l'aspect social est aussi important que l'aspect fonctionnel pour la plupart des répondants. Les aspects fonctionnels reposent essentiellement sur des comparaisons avec l'existant (bus classiques d'un système de transport). Les aspects sociaux touchent les usagers au plus profond d'eux-mêmes. Ceux qui ont utilisé la navette autonome tendent également à se projeter d'une manière générale dans le futur. Par exemple, ces derniers traitent de thèmes comme la prise en charge de l'humain par la machine. Ils évoquent enfin une certaine prise de conscience d'un changement de paradigme par rapport à l'automatisation de la mobilité. Les avis des usagers sont souvent tranchés voire même parfois contradictoires. Cela démontre beaucoup de questionnement de leur part, d'interrogation sur le futur.

En dehors des craintes, on dénote toutefois de la curiosité, un intérêt pour le progrès technologique, une fierté des usagers sondés par rapport au fait que le futur se dessine aussi à Sion, leur ville. Ils montrent également un grand intérêt écologique, économique et social. Celui-ci produit une image très positive de la ville de Sion, en tant qu'incubatrice d'idées nouvelles. De plus, durant la collecte de données, nous avons ressenti que les usagers ont envie de s'impliquer personnellement dans ce type test à l'échelle $1: 1$.

Le résultat très certainement le plus surprenant de ce test est d'avoir provoqué au sein de la population valaisanne un intense débat démocratique, qui est d'ailleurs en tout point nécessaire et crucial pour le futur de la mobilité urbaine, avec notamment des enjeux par rapport au vieillissement de la population et donc à l'importance de concevoir des moyens de transport qui prennent en compte les besoins des personnes à mobilité réduite.

\section{Conclusion}

L'analyse des résultats des enquêtes menées par la HES-SO Valais-Wallis sur la navette autonome à Sion montre l'intérêt grandissant de la population quant à l'intégration concrète des nouvelles technologies dans le quotidien des citoyens. En effet, ce projet caractérisé comme original et innovant de navette sans chauffeur donne l'image d'une ville dynamique résolument orientée vers le futur.

La navette, décrite comme compacte, discrète, écologique et facilitant la mobilité douce, représente un réel attrait touristique pour la ville. Elle permet d'accéder à des lieux moins accessibles aux voitures ainsi qu'aux bus tout en contributant à la visibilité des commerces.

Ce projet permet, d'après les répondants, de susciter une prise de conscience d'un changement de paradigme en rapport à une automatisation grandissante 


\section{Ocula ${ }^{20}$}

La place de l'usager en design

R. Ramseyer, E. Fragnière, B. Nanchen, F. Evéquoz, G. Eden I Mieux prendre ... I DOI: 10.12977/ocula2019-5

de la mobilité. Plus qu'un enjeu technologique, ce test grandeur nature a provoqué un débat démocratique sur le futur de la mobilité urbaine avec notamment des enjeux par rapport au vieillissement de la population. Notre enquête démontre l'importance de concevoir des moyens de transport qui prennent en compte les besoins des personnes à mobilité réduite. C'est certainement le résultat le plus intéressant d'autant plus qu'il provient directement des utilisateurs qui ont participé à notre étude de terrain. En effet, à l'opposé d'une production de bien, un service se doit d'être co-conçu par l'utilisateur. Intégrer l'utilisateur en amont de ce processus de design de nouveau type de service de mobilité assurera son acceptation future au sein de la société.

La navette autonome de Sion répond dès lors à des enjeux d'innovation sociale. Bien que les aspects relatifs à la sécurité restent une préoccupation majeure des citoyens, l'enquête permet de fournir des éléments de réponse pragmatiques en ce qui concerne l'amélioration du trafic, notamment en favorisant un aménagement des rues piétonnes beaucoup plus adapté. Les résultats de ce test à l'échelle $1: 1$ d'une navette autonome contribuent ainsi à une meilleure définition du plan général de la circulation de la ville tout en intégrant les nouveaux moyens autonomes de mobilité. Cette recherche qualitative sur les perceptions des utlisateurs de navette autonome n'en est qu'à ses débuts. Nous avons l'intention de poursuivre ce travail afin que l'élaboration des politiques de transport public soit basée sur des données empiriques issues des tests à l'échelle $1: 1$.

\section{Bibliographie}

Abraham, Hillary ; Lee, Chaiwoo; Brady, Samantha ; Fitzgerald, Craig ; Mehler, Bruce ; Reimer, Bryan ; Coughlin, Joseph, F.

2017 "Autonomous vehicles, trust, and driving alternatives : A survey of consumer preferences », Transportation Research Board 96th Annual Meeting, Washington, DC, <http://agelab.mit.edu/sites/default/files/private-files/ publications/2016_6_Autonomous_Vehicles_Consumer_Preferences.pdf $>$. Consulté le 02 janvier 2019.

Alessandrini, Adriano ; Campagna, Andrea ; Delle Site, Paolo ; Filippi, Francesco ; Persia, Luca

2015 « Automated vehicles and the rethinking of mobility and cities », Transportation Research Procedia, $\mathrm{n}^{\circ}$ 5. pp. 145-160.

Blomkvist, Johan,

2014 «Representing Future Situations of Service : Prototyping in Service Design », Linköping University Electronic Press, <http://www.diva-portal.org/smash/ record.jsf?pid=diva2\%3A712357\&dswid=-194>. Consulté le 5 janvier 2019.

Carr, Valerie L. ; Sangiorgi, Daniela ; Büscher, Monika ; Junginger, Sabine ; Cooper, Rachel

2011 «Integrating evidence-based design and experience-based. Approaches in healthcare service design ", HERD : Health Environments Research \& Design Journal, vol. 4, $\mathrm{n}^{\circ}$ 4, p. 12-33. 


\section{Ocula ${ }^{20}$}

La place de l'usager en design

R. Ramseyer, E. Fragnière, B. Nanchen, F. Evéquoz, G. Eden I Mieux prendre ... I DOI: 10.12977/ocula2019-5

Chan, Joel ; Fu, Katherine ; Schunn, Christian ; Cagan, Jonathan ; Wood, Kristin; Kotovsky, Kenneth

2011 " On the benefits and pitfalls of analogies for innovative design: Ideation performance based on analogical distance, commonness, and modality of examples », Journal of mechanical design, vol. 133, nº 8, p. 081004-1-11.

Creswell, John W. ; Poth, Cheryl N.

2012 Qualitative inquiry and research design: Choosing among five approaches (3rd ed.), Sage publications.

Design Council

2015 « Design methods for developing service. Guide », UK Design Council, <htpps://www.designcouncil.org.uk/sites/default/files/asset/document/ Design\%20methods\%2ofor\%2odeveloping\%20services.pdf $>$. Consulté le 8 juillet 2018.

Dubois, Michel ; Bobillier-Chaumon, Marc-Eric

2009 "L'acceptabilité des technologies : bilans et nouvelles perspectives », Le travail humain, vol. 72, no 4, p. 305-310.

Fragnière, Emmanuel ; Ramseyer, Randolf ; Grèzes, Vincent ; Kuonen, Patrick ; Nanchen, Benjamin ; Sahut, Jean-Michel

2017 "Service Design and Educational Challenges to Foster Innovation in Tourism: the case of the Swiss Matterhorn Region ", Gestion 2000, vol. 34, n 1, p. 203213.

Eden, Grace ; Nanchen, Benjamin ; Ramseyer, Randolf ; Evequoz Florian

2017 «On the road with an autonomous passenger shuttle : integration in public spaces », Proceedings of the 2017 CHI Conference Extended Abstracts on Human Factors in Computing Systems, <http://e-space.mmu. ac.uk/620059/1/On\%20the\%20Road\%20with\%20an\%20Autonomous\%20 Passenger\%20Shuttle_\%2oIntegration\%20in\%2oPublic\%2oSpaces.pdf>. Consulté le 20 janvier 2019.

Florez Rodriguez, Sergio Alberto ; Espié, Stéphane ; Nouveliere, Lydie ;

Bouaziz, Samir

2018 « Outils et méthodes pour l'étude des interactions véhicules autonomesopérateurs/passagers humains en situations critiques », HAL, <https://hal archives-ouvertes.fr/hal-01762976/document>. Consulté le 5 janvier 2019.

Hengstler, Monika ; Enkel, Ellen ; Duelli, Selina

2016 « Applied artificial intelligence and trust. The case of autonomous vehicles and medical assistance devices », Technological Forecasting and Social Change, vol. 105, p. 105-120.

Homburg, Christian ; Koschate, Nicole ; D. Hoyer, Wayne

2005 « Do Satisfied Customers Really Pay More? A Study of the Relationship between Customer Satisfaction and Willingness to Pay », Journal of Marketing, vol. 69, no 2, p. 84-96.

Mager, Birgit

2008 « Service Design », Erlhoff M., Marshall T. (eds) Design Dictionary. Board of International Research in Design. Birkhäuser Basel, pp. 354-357.

McKerracher, Colin ; Orlandi, Itamar ; Ramkumar, Surya

2016 «An integrated perspective on the future of mobility », McKinsey \& Company 


\section{Ocula ${ }^{20}$}

La place de l'usager en design

R. Ramseyer, E. Fragnière, B. Nanchen, F. Evéquoz, G. Eden I Mieux prendre ... I DOI: 10.12977/ocula2019-5

and Bloomberg New Energy Finance, <https://www.bbhub.io/bnef/ sites/4/2016/10/BNEF_McKinsey_The-Future-of-Mobility_11-10-16.pdf>. Consulté le 24 janvier 2019.

Nicolas, Jean-Pierre ; Lichère, Vincent

2016 "Véhicule autonome en milieu urbain : quelles perspectives pour les transports collectifs? Illustration à travers le projet e-vasion. Interview », Transflash, $\mathrm{n}^{\circ}$ 408, p. 1-5.

Postaouto.ch

2016 Projet «SmartShuttle »(WWW Document), <htpps://www.postauto.ch/fr/ projet-\%C2\%ABsmartshuttle\%C2\%BB-O>. Consulté le 14 décembre 2018.

Proulx, Serge

2015 "La sociologie des usages, et après? ", Revue française des sciences de l'information et de la communication, $n^{\circ} 6$, <https://journals.openedition. org/rfsic/1230>. Consulté le 10 décembre 2018.

Salonen, Arto O.

2018 « Passenger's subjective traffic safety, in-vehicle security and emergency management in the driverless shuttle bus in Finland », Transport policy 61, pp. 106-110.

Vargo, Stephen L. ; Lusch, Robert F.

2004 « Evolving to a new dominant logic for marketing », Journal of marketing 68.1, pp. 1-17.

Waytz, Adam ; Heafner, Joy ; Epley, Nicholas

2014 " The mind in the machine : Anthropomorphism increases trust in an autonomous vehicle », Journal of Experimental Social Psychology, vol. 52, p. $113-117$. 